\title{
Dielectric Relaxation Behaviour of Ethyl Methacrylate and Vinyl Acetate Grafted Poly[tetrafluoroethylene-co-(perfluorovinyl ether)] Films Prepared by Direct Radiation Grafting Method
}

\author{
Mervat Mohamed Abdel Aal, Massarat B. S. Osman, and Samia M. MokhtaR* \\ Physics Department, University College for Women, Ain Shams University, Cairo, Egypt \\ *Chemistry Department, University College for Women, Ain Shams University, Cairo, Egypt
}

(Received June 12, 1995)

\begin{abstract}
Dielectric relaxation of poly(tetrafluoroethylene-co-perfluorovinyl ether) (PFA) grafted by ethyl methacrylate (EMA) and vinyl acetate (VAc) of different compositions prepared by direct radiation was studied at $0.1,1,10$, and $100 \mathrm{kHz}$ between 273 and $550 \mathrm{~K}$. Two well resolved relaxation peaks are observed in all films except that with EMA/VAc of compositon $10 / 90 \mathrm{wt} \%$ in which the second peak is masked. In PFA film grafted by EMA/VAc of 50/50 wt \% at low temperature the third relaxation was observed. The activation energy for the relaxation was determined from Arrhenius plots. The ac conductivity of the films was investigated and the activation energy of conduction was evaluated. X-Ray diffraction patterns and IR spectra were also measured.

KEY WORDS Dielectric Constant $\varepsilon^{\prime} /$ Dielectric Loss $\varepsilon^{\prime \prime} /$ Conductivity / Activation Energy /
\end{abstract}

Measurement of dielectric loss $\left(\varepsilon^{\prime \prime}\right)$ and relative permittivity as functions of frequency and temperature is a well established method of detecting molecular motion in macromolecules, particularly in the solid state. Amorphous polymers may exhibit five types of relaxation regions $^{2}$ : (1) motion of the entire chain as a unit; (2) motion of a chain segment of perhaps $50-100$ carbon atoms, i.e., glass transition; (3) motion of a 2, 3, or 4 carbon atom moiety about the chain axis; (4) motion of a side group about an axis at right angles to the chain, and (5) very low temperature loss which may or may not be the same as (4). Not all relaxation processes are observed in dielectric experiment. A process is dielectrically active only if it involves the reorientation of the dipole-moment vector. ${ }^{3}$

The dielectric properties of flexible polar side-amorphous polymers depend on the position and direction of the dipolar group with respect to the chain backbone. Two main kinds of relaxation are generally observed in this type of polymer. A high temperature relaxation $(\alpha)$ associated with chain backbone motion and a low temperature relaxation $(\beta)$ which results from the side group motion. ${ }^{4,5}$

Even in amorphous polymers without side groups, such as amorphous polyesters, ${ }^{6}$ two kinds of relaxation are also observed. The molecular mechanism of the hightemperature relaxation is also the large-scale conformational rearrangement of the main chains; the low-temperature relaxation is due to the local twisting modes of main chains when the large-scale conformational rearrangement of main chain is frozen. ${ }^{7-9}$ This is also called $\beta$ relaxation since it has characteristics similar to the $\beta$ relaxation in polymers with flexible polar side groups, both originate in local motions. ${ }^{10}$

Relaxation of methacrylate polymers has been the object of many studies. ${ }^{11-13}$ The dielectric behaviour of poly(vinyl ester) is of particular interest to many authors. $^{15-17}$ In poly(vinyl acetate) (PVAc) two relaxation regions, $(\alpha)$ and $(\beta)$, are observed. ${ }^{14,15}$ In this study, the dielectric relaxation of poly(tetrafluoroethylene-co- perfluorovinyl ether) (PFA) film grafted with different compositions of ethyl methacrylate and vinyl acetate comonomer is investigated.

\section{EXPERIMENTAL}

Poly(tetrafluoroethylene-co-perfluorovinyl ether) (PFA) film of thickness $50 \mu \mathrm{m}$ (Mitsui Fluoro Chemical Co., Ltd., Japan) was used. PFA was washed with acetone and dried in a vacuum oven at room temperature. Vinyl acetate (Merck) and ethyl methacrylate (Merck) of purity $99 \%$ were used. The other chemical reagents were used as received.

The direct radiation grafting method was used in a nitrogen atmosphere. A glass ampoule containing the monomer solutions in ethyl acetate as diluent and film sample was deareated by bubbling nitrogen for 5-7 min, sealed and then subjected to $\mathrm{Co}^{60} \gamma$-radiation at a dose rate of $0.2 \mathrm{Gys}^{-1}$. The grafted film was washed thoroughly with hot acetone and soaked overnight in acetone in order to extract any residual monomers or homopolymers possibly included in the film. This process was also repeated using ethyl alcohol. The grafted film was then dried in a vacuum oven at $60^{\circ} \mathrm{C}$ for $24 \mathrm{~h}$ and weighed. The degree of grafting $(G \%)$ was determined by the percentage increase in weight: $G \%=100$ $\left(W_{\mathrm{g}}-W_{\mathrm{G}}\right) / W_{\mathrm{o}}$, where $W_{\mathrm{o}}$ and $W_{\mathrm{g}}$ represent the weight of original and grafted films, respectively.

IR spectra were obtained for all grafted films using a Perkin-Elmer 1430 Ratio Recording Infrated Spectrophotometer. X-Ray patterns were determined for all films with Phillips apparatus (pw 1390 channel control and pw 1373 goniometer supply) using nickel filtered $\mathrm{Cu}-K_{\alpha}$ radiation.

Measurements of dielectric constant $\left(\varepsilon^{\prime}\right)$ and loss tangent $(\tan \delta)$ were made with a model SR 720 LCR meter which can be operated at 5 frequencies of the applied potential ranging from 0.1 to $100 \mathrm{kHz}$. Frequencies are accurate to $0.01 \%$ and error associated with $\tan \delta$ is approximately \pm 0.0001 . Furnace capability 
permitted measurement from 273 to $550 \mathrm{~K}$. Temprature was controlled to $\pm 0.5^{\circ} \mathrm{C}$.

To use the sample as dielectric medium in a parallelplate capacitor, different electrodes were tested. Silver in the form of a silver emulsion paint in which the silver is suspended in a fast-drying solvent was used.

\section{RESULTS AND DISCUSSION}

\section{Effects of Comonomer Composition}

Table I shows the effects of comonomer composition on the degree of grafting onto poly(tetrafluoro ethylene-co-perfluoro vinyl ether) (DFA) films in the presence of ethyl acetate as a diluent. It is obvious that the degree of grafting increases as the content of VAc in the binary monomers decreases to reach a maximum for comonomer composition of $\sim 20 / 80 \mathrm{wt} \%$ of VAc/EMA. Thereafter, at higher conent of EMA the degree of grating falls to the lowest value for $100 \mathrm{wt} \%$ EMA.

These results indicate that the presence of EMA may increase the diffusion of both monomers onto the polymer substrate. The homopolymerization process of both VAc and EMA is thus reduced. This leads to enhancement of grafting and a higher grafting yield

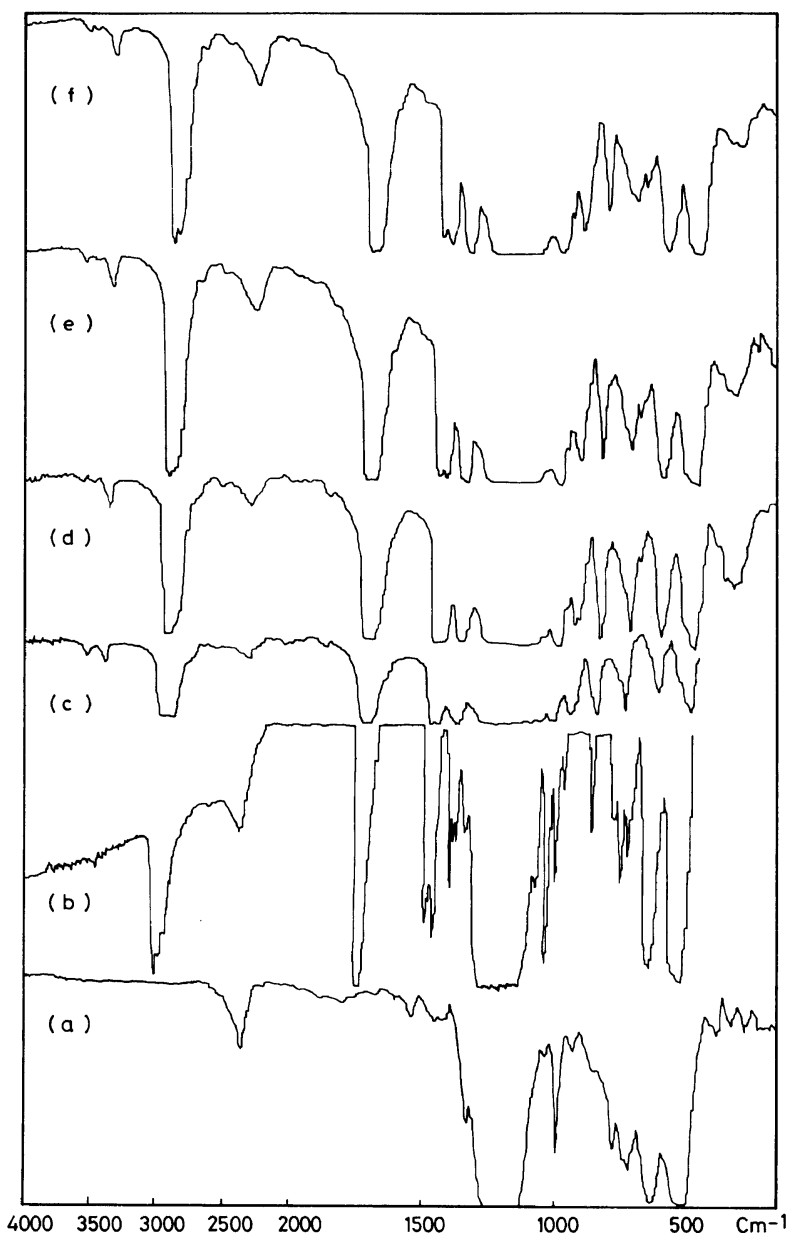

Figure 1. IR spectra of original and grafted PFA films having different degrees of grafting: (a) original PFA film; (b) PFA- $g$-PEMA; (c) PFA- $g$-P(EMA/VAc) of composition $80 / 20 \mathrm{wt} \%$; (d) PFA- $g$ P(EMA/VAc) of composition 40/60 wt \%; (e) PFA- $g$-P(EMA/VAc) of composition 20/80 wt $\%$; and (f) PFA- $g$-P(EMA/VAc) of composition $10 / 90 \mathrm{wt} \%$ was obtained. However, with increasing content of EMA in the binary system ( $>80 \mathrm{wt} \%$ EMA) growing radicals of EMA may enhance the termination rate and hence, final grafting yield is lowered.

\section{IR-Spectra}

Figure 1 shows the spectra of the original and grafted

Table I.

\begin{tabular}{|c|c|c|c|c|c|c|c|}
\hline \multirow{3}{*}{$\frac{(\mathrm{EMA} / \mathrm{VAc})}{w \mathrm{t} \%}$} & \multirow{3}{*}{$\frac{\begin{array}{c}\text { Degree of } \\
\text { grafting }\end{array}}{G^{\%} \%}$} & \multirow{3}{*}{$\varepsilon_{\mathrm{r} . \mathrm{t} .}^{\prime}$} & \multicolumn{4}{|c|}{$s$} & \multirow{3}{*}{$\frac{W}{\mathrm{eV}}$} \\
\hline & & & \multirow{2}{*}{$\frac{273}{K}$} & \multirow{2}{*}{$\frac{280}{\mathrm{~K}}$} & \multirow{2}{*}{$\frac{290}{K}$} & \multirow{2}{*}{$\frac{300}{\mathrm{~K}}$} & \\
\hline & & & & & & & \\
\hline $100 / 0$ & 25.9 & 3.24 & 0.99 & 0.97 & 0.92 & 0.85 & 0.58 \\
\hline $90 / 10$ & 240 & 3.60 & 0.95 & 0.93 & 0.92 & 0.85 & 0.67 \\
\hline $80 / 20$ & 266 & 3.30 & 0.85 & 0.80 & 0.80 & 0.75 & 0.63 \\
\hline $60 / 40$ & 156.9 & 3.15 & 0.75 & - & 0.75 & 0.7 & 0.39 \\
\hline $50 / 50$ & 144.9 & 3.20 & - & - & - & - & - \\
\hline $40 / 60$ & 132.9 & 4.30 & 0.70 & 0.85 & 0.70 & 0.65 & 0.77 \\
\hline $20 / 80$ & 170.5 & 3.20 & 0.75 & 0.70 & 0.65 & 0.60 & 1.06 \\
\hline $10 / 90$ & 50.6 & 1.0 & 0.66 & 0.64 & 0.62 & 0.60 & 0.58 \\
\hline $0 / 100$ & 37.7 & 2.35 & 0.60 & 0.58 & 0.57 & 0.56 & 0.20 \\
\hline
\end{tabular}

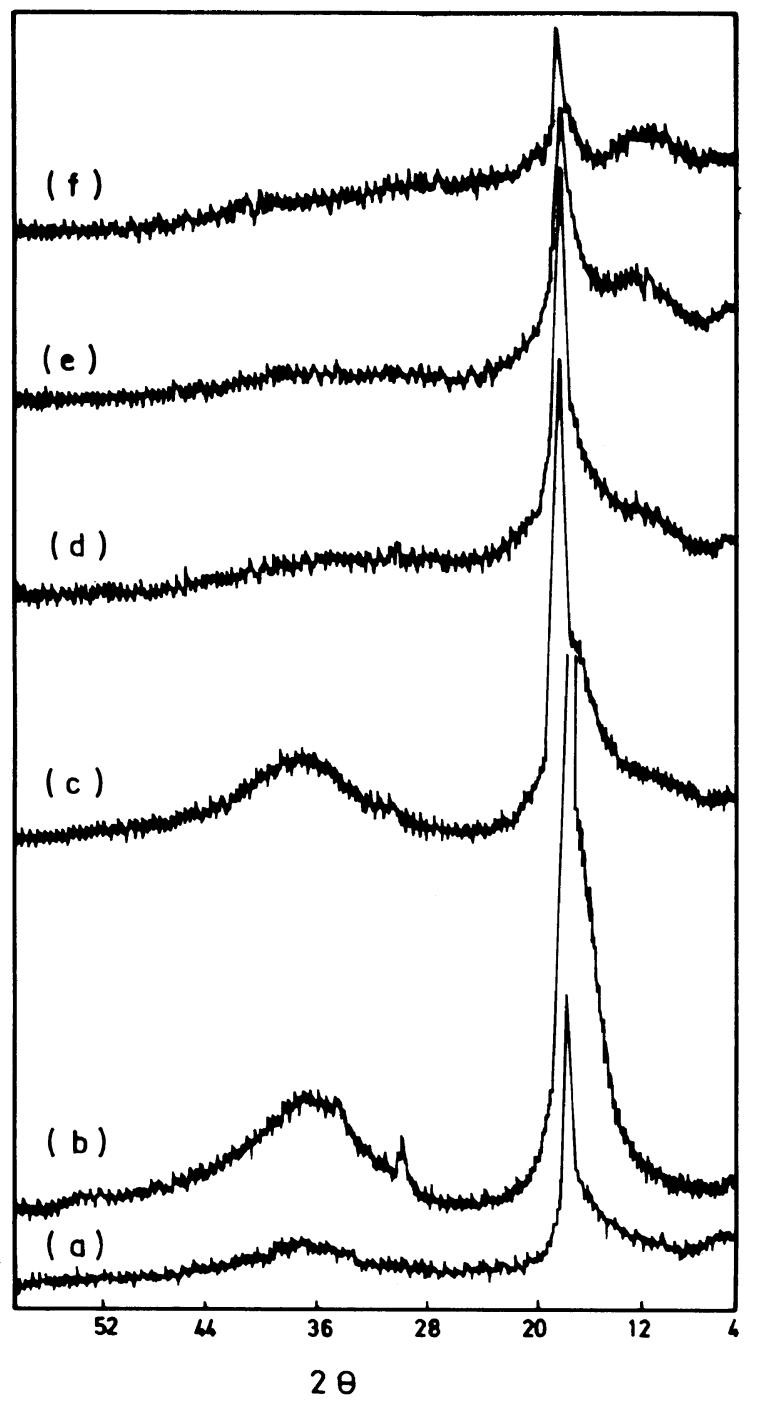

Figure 2. X-Ray diffraction patterns of the original PFA film and graft copolymers: (a) PFA-g-PVAc; (b) original PFA film; (c) PFA- $g$-PEMA; (d) PFA- $g$-P(EMA/VAc) $(40 / 60)$; (e) PFA- $g$-P(EMA/VAc) $(60 / 40)$ and (f) PFA- $g$-P(EMA/VAc) $(80 / 20)$. 
PFA films having different degrees of grafting. The characteristic band of PVAc and PEMA graft chains appear clearly in the spectra at $1700-1740 \mathrm{~cm}^{-1}$, confirming the existence of carbonyl groups of both vinyl acetate and ethyl methacrylate. The intensity of this band increases as the degree of grafting increases. So the degree of grafting can be followed from the intensity of this band. The absorption which appeared at $2900-3000 \mathrm{~cm}^{-1}$ was assigned to $-\mathrm{CH}_{2}$ asymmetric stretching. ${ }^{18}$

\section{$X$-Ray Diffraction}

Figure 2 illustrates the X-ray diffraction of the original PFA film and films grafted with PVAc and PEMA and films grafted by different compositions of EMA and VAc. As seen from Figure 2b, for the original PFA, a strong reflection at $2 \theta=18^{\circ}$ and two weaker reflections at $2 \theta=31.6^{\circ}$ and $36.8^{\circ}$ appear. These reflections are measures of the crystalline phase in the PFA film. A broad reflection at $2 \theta=39.2^{\circ}$ is also seen in PFA pattern. This pattern is associated with the amorphous phase in the sample. The sharp and broad reflections indicate semicrystallinity. ${ }^{18}$ The X-ray patterns of the grafted films with PVAc and PEMA are given in Figures $2 \mathrm{a}$ and $2 c$, respectively. These patterns differ from that given for
PFA. The intensity of the reflection ascribed to the crystalline phase of PFA decreases in Figures $2 \mathrm{a}$ and c. ${ }^{19}$ The reflections at $31.6^{\circ}$ and $36.8^{\circ}$ disappear completely in the pattern of the film grafted with PVAc (Figure 2a). This is correlated with the higher $G \%$ with amorphous PVAc than with PEMA (Table I). Figures 2d, e, and $\mathrm{f}$ show the patterns for the PFA films grafted with $40 / 60$, $60 / 40$, and $80 / 20 \mathrm{wt} \%$ of EMA/VAc, respectively. This figure shows that the pattern of all films exhibit one or two diffuse halos. This indicates that all films had essentially amorphous morphology. ${ }^{19}$ The intensity of the crystalline reflection at $2 \theta=18^{\circ}$ decreases gradually by increase of grafting, indicating increase of the amorphous phase in the grafted films. The less intensive reflections at $31.6^{\circ}$ and $36.8^{\circ}$ disappear completely in Figures $2 \mathrm{~d}$, e, and $\mathrm{f}$. The halo at $2 \theta=39.2^{\circ}$ decreases in intensity while a new halo appears at $2 \theta<15$ indicating the amorphous phase of the PEMA/PVAc copolymers. The intensity of this halo increases to maximum intensity for the highest grafting film (80/20, Figure 2d). In this film, the intensity of the reflection at $18^{\circ}$ decreases to $30 \%$ its value for the original PFA.

\section{Dielectric Relaxation}

The temperature dependence of the dielectric con-

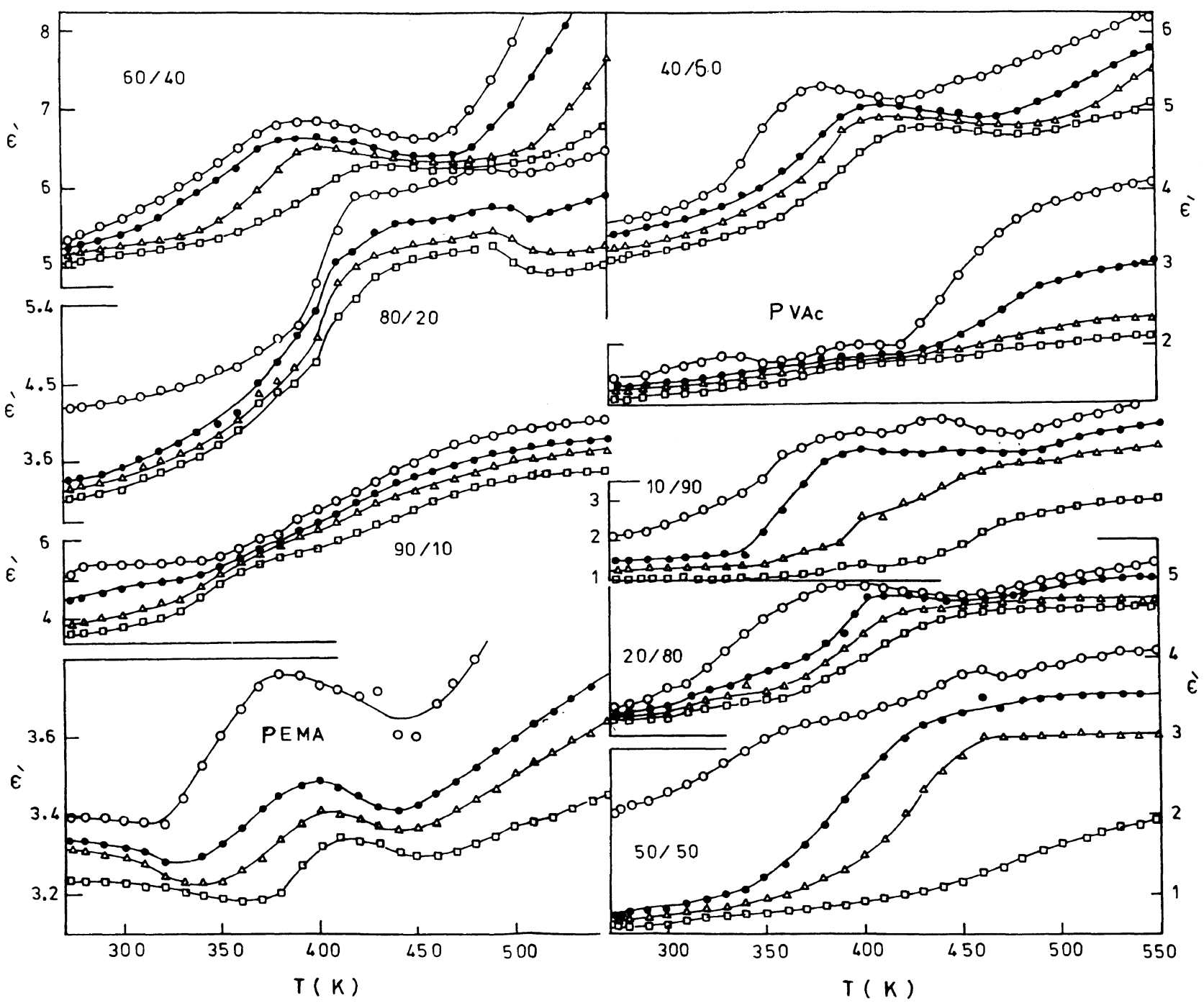

Figure 3. Temperature dependence of the dielectric constant $\left(\varepsilon^{\prime}\right)$ for PFA grafted films at: $(\bigcirc) 0.1 ;(\bigcirc) 1.0 ;(\triangle) 10.0 ;(\square) 100 \mathrm{kHz}$. 
stant $\left(\varepsilon^{\prime}\right)$ of the investigated PFA films grafted with EMA-VAc with different compositions and different grafting $\%$ is shown in Figure 3. This figure shows the following general trends in the dielectric data for all films:

1. The grafted films differ in their dielectric constant at any given frequency and temperature. Table I gives $\varepsilon^{\prime}$ values in $0.1 \mathrm{kHz}$ at room temprature $\left(\varepsilon_{\text {r.t. }}^{\prime}\right)$ for all samples.

2. $\varepsilon^{\prime}$ is inversely dependent upon frequency at all temperatures.

3. Except for the lowest frequency measurements $(100 \mathrm{~Hz})$, the dielectric constant remains almost independent of temperature up to $325-340 \mathrm{~K}$ and thereafter increases with temperature. Such increase in $\varepsilon^{\prime}$ may be expected to occur above the glass transition temperature $\left(T_{\mathrm{g}}\right)$.

4. Measured dielectric constants, ranging from 0.1 to 9.0 , are low.

5. Increasing the ratio of EMA in the grafted films up to $60 \mathrm{wt} \%$ increases $\varepsilon_{\text {r.t. }}^{\prime}$ at any frequency.

6. Increase in $\varepsilon^{\prime}$ with temperature reaches higher values as the frequency is decreased. The average increase in $\varepsilon^{\prime}$ by heating to $550 \mathrm{~K}$ is most obvious in films grafted by $80 / 20$ and $90 / 10 \mathrm{wt} \%$ at all frequencies. These two films have similar compositions and similar high grafting $\%$. This indicates that $\varepsilon^{\prime}$ may depend on either the composition of the film and/or its grafting $\%$ on the PFA film.

7. In film grafted by EMA and by $60 / 40 \mathrm{wt} \%$, increase in $\varepsilon^{\prime}$ at higher temprature and lower frequency is quite marked. This indicates the formation of a boundary layer of blocked ions at the electrode surface. The absence of increase of $\varepsilon^{\prime}$ in the rest of the grafted films means that this increase cannot be attributed to the silver electrode effect. The reason for the increase in $\varepsilon^{\prime}$ on using a silver electrode is believed to be that the silver electrode material is in fact a composite of silver and polymeric material. Large local concentrations of the latter can lead to insulating regions on the electrode surface. $^{20}$

In Figure 4, the dielectric loss tangent $(\tan \delta)$ of the investigated PFA grafted films is given as a function of temperature between 273 and $550 \mathrm{~K}$ at $10 \mathrm{kHz}$. These curves are qualitatively similar to those obtained at 0.1 , 1 , and $100 \mathrm{kHz}$. Figure 4 reveals the existence of two well resolved relaxations in all investigated films except that grafted with $90 / 10$. A sharp upturn in $\tan \delta$ is observed at some temperatures above that of the curve maximum. This is seen in film grafted by EMA and 40/60 and is less in film grafted by $80 / 20$. This effect derives from ionic impurities. The ionic conduction gives rise to its own electric loss and the effect is particularly strong as the polymer passes through $T_{\mathrm{g}} \cdot{ }^{21}$ This increase in $\tan \delta$ is less in films grafted by $50 / 50$ and $10 / 90$.

When two phases are present, at temperatures above the first $T_{\mathrm{g}}$, dc loss in the lower temperature phase can swamp the loss which is attributed to the dipole motion, thus preventing observation of the upper temperature loss peak. This explains the disappearance of the two loss peaks in the film grafted by $10 / 90$. Since there is no capacitive component associated with unimpeded ion transport, the appearance of the two loss

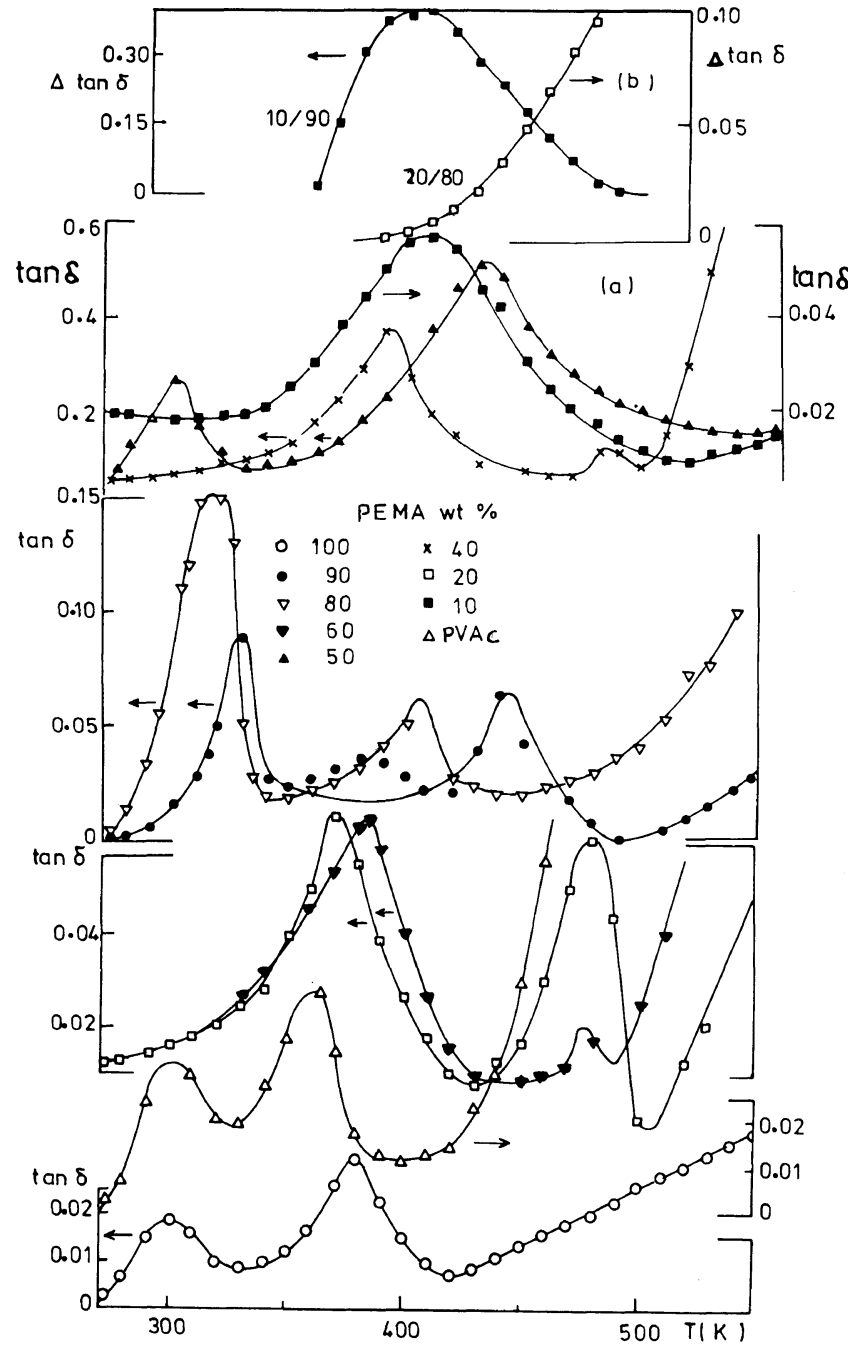

Figure 4. (a) Tan $\delta$-temperature dependence for all grafted films at $10 \mathrm{kHz}$. (b) $\Delta \tan \delta$-temperature dependence for PFA- $g$-P(EMA/VAc) of composition $10 / 90 \mathrm{wt} \%$ and $20 / 80 \mathrm{wt} \%$.

peaks in $\tan \delta$-temperature dependence may be considered evidence that all the rest of the samples form a single phase.

Since it appears that the peak value and temperature of the loss tangent are dependent on frequency, the composition and/or grafting $\%$ of the films, dielectric loss $\left(\varepsilon^{\prime \prime}\right)$ values have been calculated for the data of $\tan \delta$ and $\varepsilon^{\prime}$. Since the frequency dependence of $\varepsilon^{\prime \prime}$ at different temperatures exhibits broad peaks while the temperature dependence of $\varepsilon^{\prime \prime}$ gives curves with sharp peak, $\varepsilon^{\prime \prime}-T$ dependence is given in Figure 5 for all films at different frequencies of measurement. These curves reveal the existence of two well resolved relaxations in all investigated films except that grafted with $10 / 90$. The location and strength of the relaxation were determined from these figures. Both the maximum values of the dielectric loss $\left(\varepsilon_{\mathrm{m}}^{\prime \prime}\right)$ and the position of these relaxations $\left(T_{\mathrm{m}}\right)$ are different for different films. The values of $\varepsilon_{\mathrm{m}}^{\prime \prime}$ and $T_{\mathrm{m}}$ are dependent on frequency. The low-temperature relaxation is associated with local molecular motion, due to hindered rotation of the ethyl acrylate group or the acetate group about the $\mathrm{C}-\mathrm{C}$ bond linking it to the chain backbone. Hereafter, this peak is called the $\beta$ relaxation. The higher-temperature loss, 


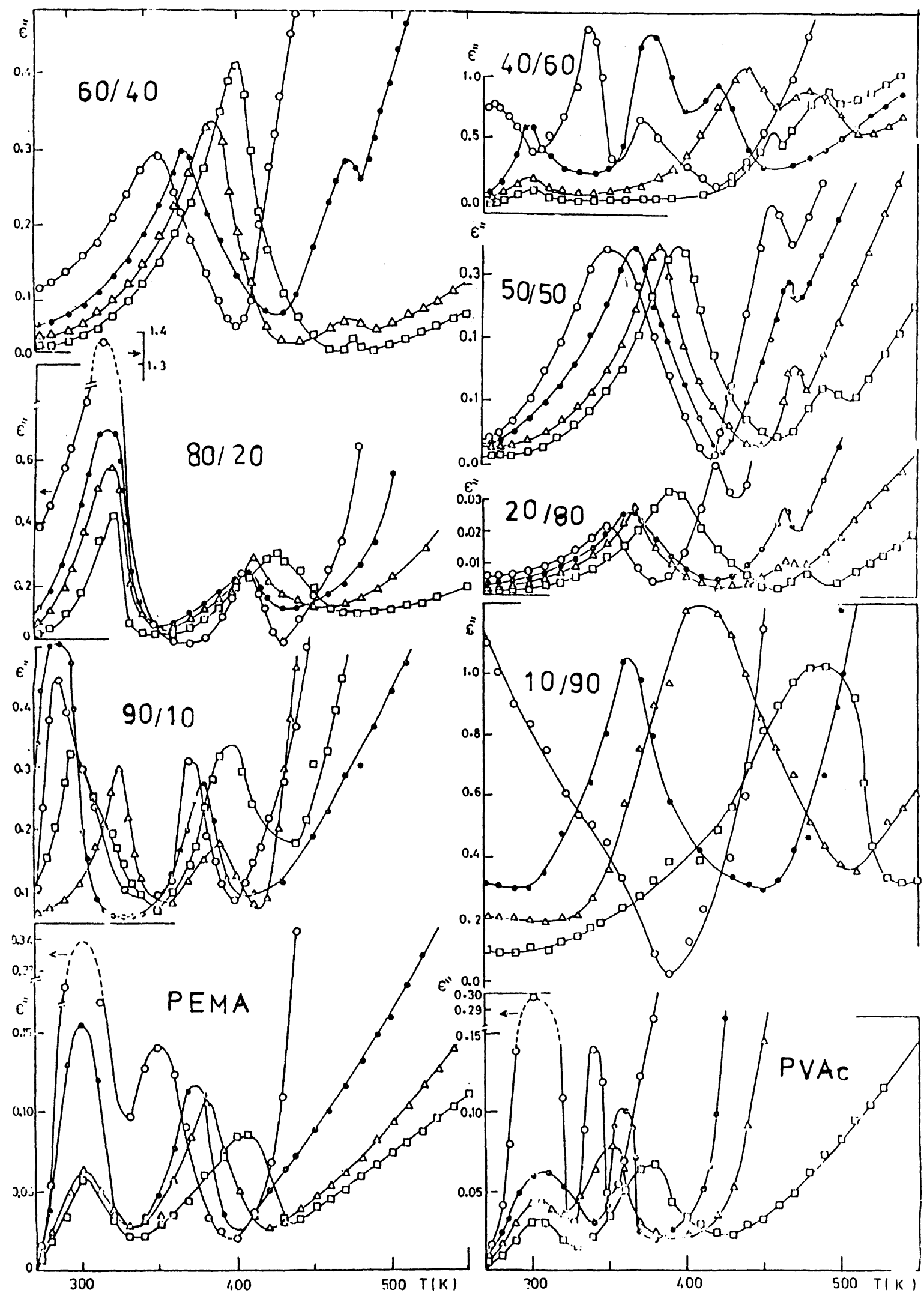

Figure 5. Dielectric loss $\left(\varepsilon^{\prime \prime}\right)$-temperature dependence for the grafted PFA films at: $(O) 0.1$;

$1.0 ;(\triangle) 10.0 ;(\square) 100 \mathrm{kHz}$

referred to as the $\alpha$-relaxation, is associated with the chain backbone motion. ${ }^{1-5}$

In film grafted by $(50 / 50)$, the third peak (hereafter called $\gamma$ peak) appears clearly separated from the $\beta$ peak on the low-temperature side. This $\gamma$-transition temperature is very reducible at all frequencies using different pieces of film. In this region, the main backbone chains are effectively "frozen in." The apparent third process $(\gamma$-peak $)$ in the $50 / 50$ film is associated with additional segment motion faster than those of the $\beta$ process, that occurs in all samples. The disappearance of this third peak in other films indicates that the intermolecular 
potential in these samples is high enough to reduce either the number of molecular segments whose motion can be seen as a peak and/or cause them to completely freeze out within the time of measurement. ${ }^{22,23}$

Both $\beta$ and $\alpha$-relaxations shift to lower temperature as frequency decreases. A change in the shape of the low- and high-temperature peaks is evident in Figure 5. This is seen either as a change in the rate of change of $\varepsilon^{\prime \prime}$ with temperature on the low temperature side of the peak, as the composition of the monomer and or its grafting $\%$ change, or as a change in the width at half height of the peak. This width decreases to $10 \mathrm{~K}$ for the $\beta$-peak of the highest grafting $\%$ film $(90 / 10)$ and increases to $75 \mathrm{~K}$ for the lowest grafted film (10/90). This width is nearly constant $(\sim 60 \mathrm{~K})$ for films grafted by $40 / 60$. The increase of the half width at half height of the peak in film grafted by $10 / 90$ indicates either an increase in the distribution of relaxation time of the films and/or a second relaxation peak at lower temperature masked by a relatively large contribution to $\varepsilon^{\prime \prime}$ from the higher temperature $\beta$ peak. ${ }^{24}$ To determine which of the two effects dominates $\tan \delta$ and therefore $\varepsilon^{\prime \prime}$ of these films and wheather or not a second relaxation occurs in these films, the difference, $\Delta \tan \delta$, between the $\tan \delta$ values measured at 1 and $10 \mathrm{kHz}$, against temperature is plotted for the film grafted by 10/90 in Figure $4 \mathrm{~b}$. As described by Chung et al., ${ }^{25}$ if $\Delta \tan \delta-T$ plot exhibits a peak, or a shoulder, another relaxation is pesent, but if this plot shows a rapid decrease of $\Delta \tan \delta$ with decreasing temperature, no relaxation is present. From Figure $4 \mathrm{~b}$ it is observed that $\Delta \tan \delta$ shows a peak for the film grafted with $10 / 90$. Therefore a second relaxation exists at this sample, but a large contribution to $\tan \delta$ from the high-temperature peak masks this second peak. The position of this second peak in the loss tangenttemperature dependence cannot be easily resolved. In Figure $4 \mathrm{~b}$ a sharp decrease in $\Delta \tan \delta$ is observed as temperature decreases for $20 / 80$ film for which $\Delta \tan \delta$ becomes almost zero at near $380 \mathrm{~K}$. Therefore, no other relaxation exists in this film. This film is chosen as it is grafted with an intermediate composition between the film grafted by $10 / 90$ where only one relaxation peak appears and the film grafted by $50 / 50$ in which three relaxation peaks are observed.

The $\beta$-relaxation for PFA film grafted by EMA occurs between $298 \mathrm{~K}$ at $0.1 \mathrm{kHZ}$ and $303 \mathrm{~K}$ at $100 \mathrm{kHz}$. Temperature obviously increases as EMA decreases in the grafted films. This continues to approximately $60 / 40$ where the loss peak temperature then remains constant at $348 \mathrm{~K}$ for $0.1 \mathrm{kHz}$ and $396 \mathrm{~K}$ for $100 \mathrm{kHz}$ independent of any further increase of PVAc. This may be due to the fact that the main amorphous relaxation in the EMA-VAc copolymrers in the grafted films is a long and cooperative process. However, at some critical VAc concentration, this changes and the principle moving groups become isolated polymeric units of vinyl acetate.

The frequency for maximum $\varepsilon^{\prime \prime}$ is taken from $\varepsilon^{\prime \prime}-T$ curves at different frequencies and plotted against reciprocal temprature of maximum the dielectric loss $\left(1 / T_{\mathrm{m}}\right)$ in Figure 6 to obtain the activation energies of $\alpha$ - and $\beta$-relaxations. However, plots of the $\alpha$-relaxation for some films are curved and cannot be described by a

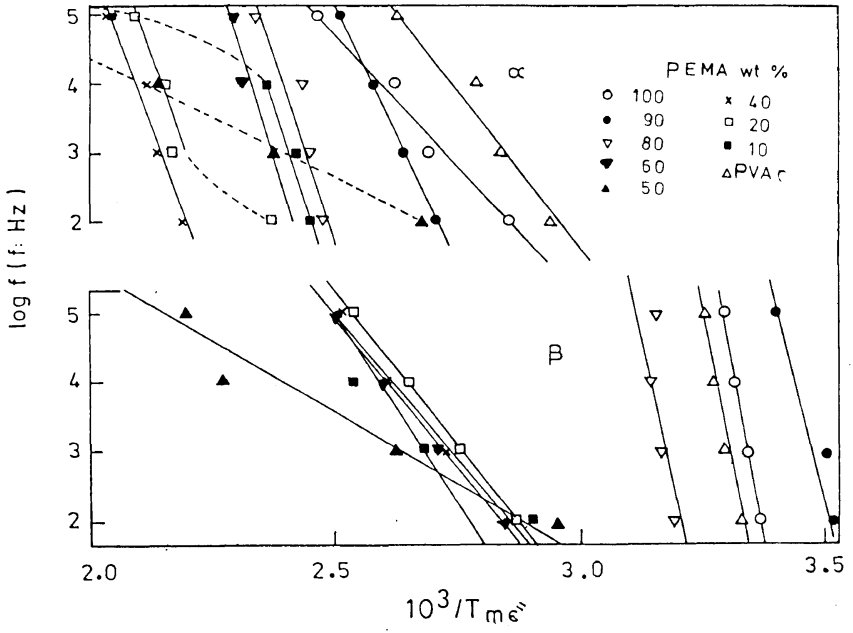

Figure 6. Arrhenius plots for the $\alpha$ and $\beta$ relaxations for all grafted films.

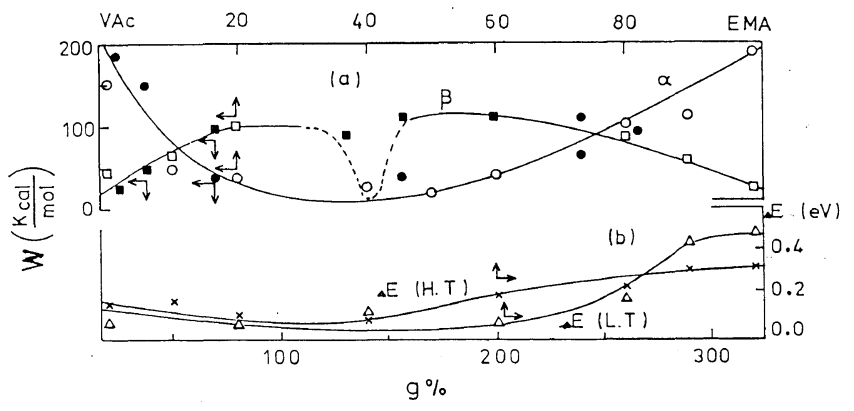

Figure 7. (a) Activation energy of $\alpha$ relaxation: ( $\square$ ) versus composition and ( $\square$ ) versus grafting \%. Activation energy of $\beta$ relaxation $(\bigcirc)$ versus composition and $(\bigcirc)$ versus grafting \%. (b) Activation energy of conduction of the films versus composition at $0.1 \mathrm{kHz}$ : $(\triangle)$ at low temperature range and $(x)$ at high temperature range.

single activation energy. This non-Arrhenius behavior is typical of amorphous polymers. ${ }^{1}$ The mean average energy for the two relaxations is plotted in Figure 7 as a function of the composition and grafting \%. The activation energy of the $\beta$ process of film grafted by VAc or EMA is maximum. Its value decreases as the second monomer is added to reach a minimum for 50/50 film. The activation energy of the relaxation of the film grafted by VAc or EMA is minimum and increases as grafting $\%$ increases or as the second monomer, either EMA or VAc, increases. This indicates that the dipolar orientation is more restricted in the films grafted by PEMA-PVAc than that grafted by PEMA or PVAc. The activation energy of this relaxation becomes maximum for film grafted by $60 / 40$. Film grafted by $50 / 50$ shows an obvious drop in activation energy as expected.

To clarify the effects of grafting of PFA with the PVAc and PEMA of different compositions on the conduction of the PFA films, the logarithm of the ac conductivity $\left(\sigma_{\mathrm{ac}}\right)$ of the samples was studied as a function of $1 / T$ for four fixed frequencies $(0.1,1,10$, and 100 $\mathrm{kHz}$ ) at $273-500 \mathrm{~K}$. The general behavior of the $\sigma_{\mathrm{ac}}-T$ curves is very similar. The plot shows two linear dependences in the low and high temperature ranges (Figure 8 ). The only simple relationship that could be used to describe the functional dependence on temperature was $=\sigma_{0} \exp (-W / K T)$ where $W$ was slightly frequency dependent. The value of the activation energy $W$ at the 


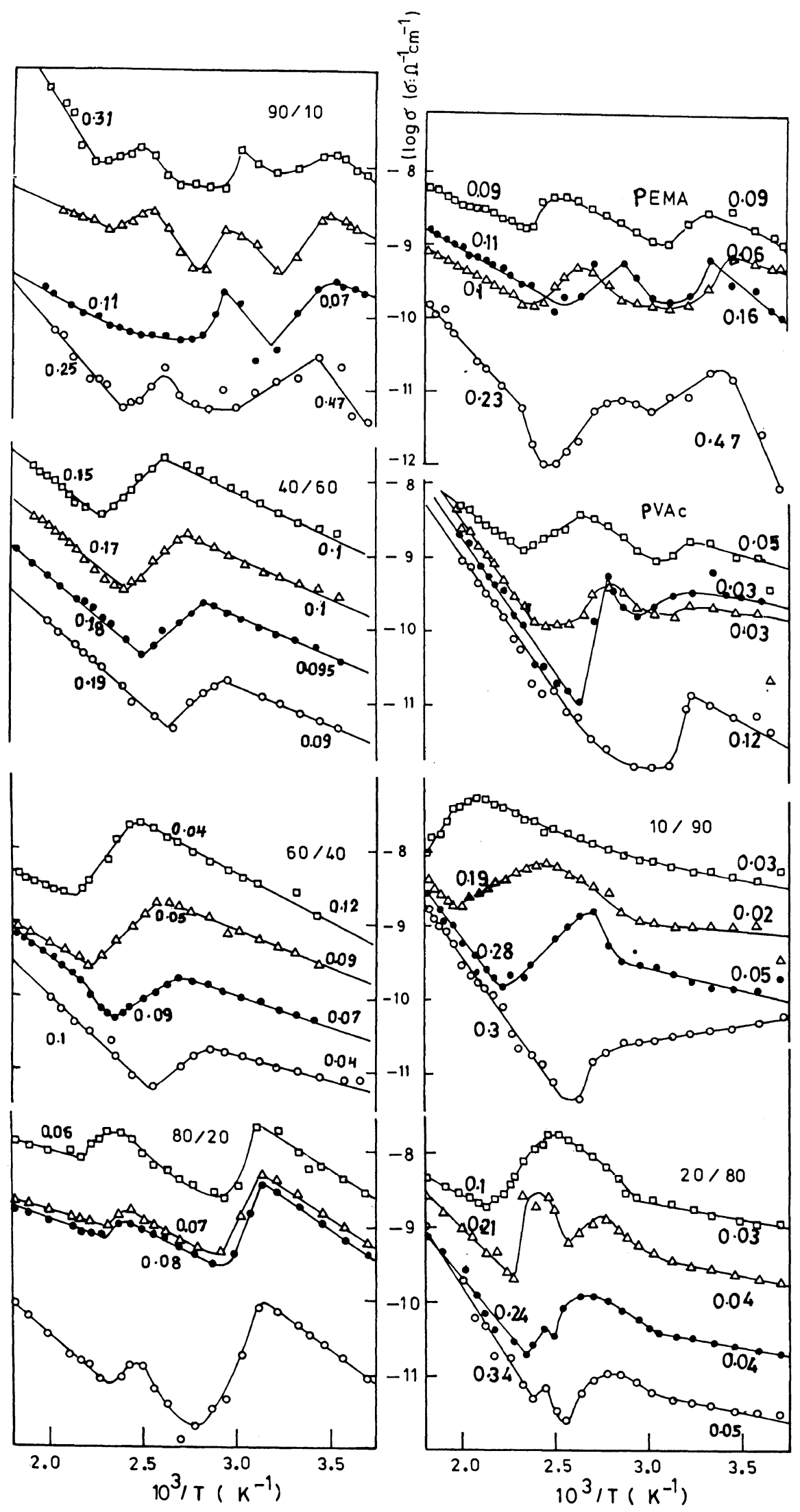

Figure 8. $\log \sigma_{\mathrm{ac}}(1 / T)$ for PFA grafted films: (○) $0.1 ;(\bullet) 1.0 ;(\triangle) 10.0$; $(\square) 100 \mathrm{kHz}$.

low and high temperature at different frequencies for all samples is written in $\mathrm{eV}$ in Figure 8 and is presented as a function of the composition of the comonomer in Figure 7 at $0.1 \mathrm{kHz}$. The sample with $60 / 40$ has minimum activation energy in the high temprature range. That with
10/90 has minimum activation energy at low temperature range. It was usually possible to reproduce within about $10 \%$ the bulk value of the conductivity when using different samples cut from the same film. The ac conductivity is higher than the dc conductivity. Dc 


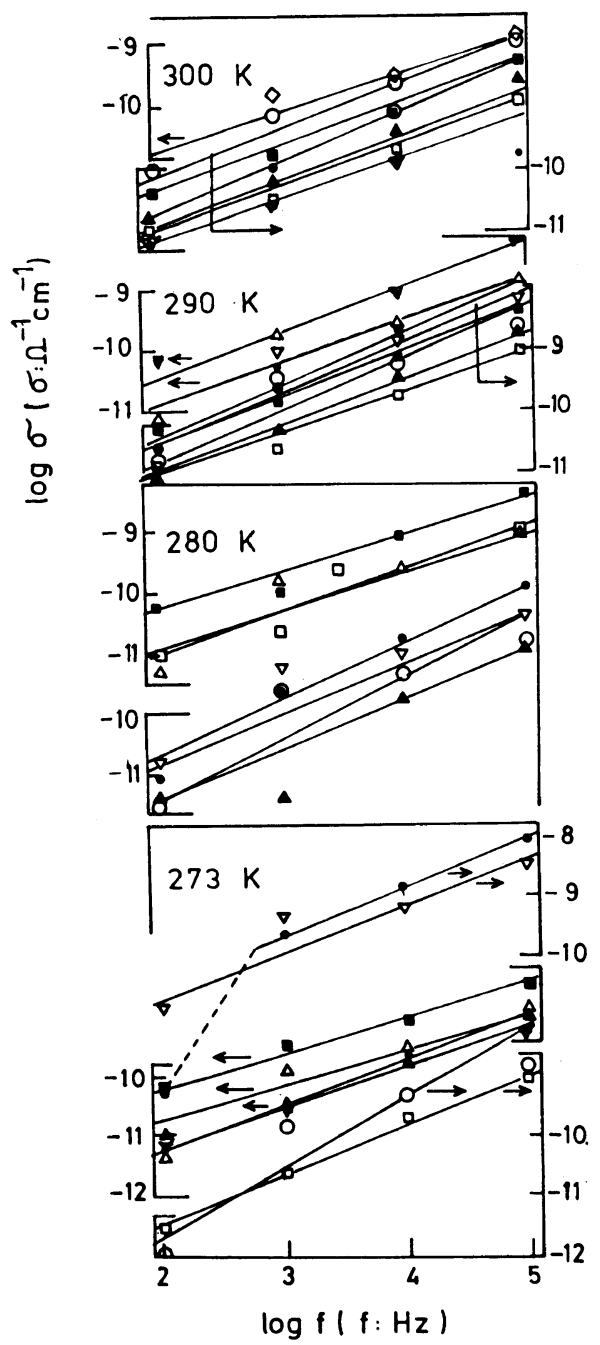

Figure 9. $\log \sigma_{\mathrm{ac}}-\log f$ dependence at different temperatures for the grafted films; PFA-g-P[EMA/VAc] of composition (wt $\%):(\bigcirc)$

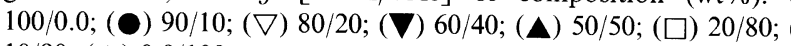

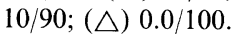

conductivity was difficult to determine at low temperature because of the experimental difficulty of measuring current less than $10^{-14} \mathrm{~A}$. At higher temperature, the temperature dependence of the conductivity becomes strong and variation of the conductivity with frequency becomes small for most films (Figure 8). Dc conductivity was always less than the ac conductivity up to the highest temperature of measurement.

At low temperature, the frequency dependence of the ac conductivity is,

$$
\sigma_{\mathrm{ac}}=A W^{s}
$$

where $A$ and $s$ are weakly temperature dependent parameters and characteristic of the mode of conduction. $A$ and $s$ are obtained from the experimental $\log \sigma_{\mathrm{ac}}-\log f$ curves and $(f w=2 \pi t)$ is the measuring frequency (Figure 9). For many amorphous solids, e.g., organic polymers, silicon, and aluminum oxides, $s$ has a value in the range $1.1-0.5$ and frequency less than unity. ${ }^{26-28}$ The values of the exponent $(s)$ estimated from the slopes of $\log$ ac vs. $\log f$ at different temperature are given in Table I. The values of $(s)$ decrease with decreasing PEMA concentration in the comonomer. The high values of the exponent corresponding to measurements made at low temperatures for instance decrease from 0.99 to 0.56 (when temperature increases from 273 to $350 \mathrm{~K}$ ). The variation in the exponent $s$ reflects the change in the nature of the conduction process with change in temperature and composition. Thus, a free carrier model is not operative in low temperature regions in the grafted PFA films. This characteristic feature is well known in low mobility amorphous systems and even in crystalline materials and has been attributed to the distribution of relation time arising from local disorder. ${ }^{29,30}$

To account for the temperature and frequency dependence of ac conductivity, various theoretical models appear in literature. ${ }^{29,31}$ The hopping model proposed by Pike considers the hopping of the carriers between two sites over a barrier separating them, rather than quantum mechanical tunneling through the barrier. The distribution of relaxation time for this process arises from the exponent dependence of relaxation time on barrier height. A feature of this model is that the values of $s$, in excess of the maximum value of 0.8 permitted within the limit of the tunneling model, are readily obtainable. In addition, $s$ is predicted to have temperature dependence and the magnitude of $s$ at any temperature is determined by the binding energy $W$ of the carrier. The polaring activation energy ${ }^{31}$ is given by $W=6 K T /(1-s)$.

The values of $s$ at different temperatures and of $W$ are given in Table I. The values of $W$ were highest for highly grafted film with EMA/VAc of 20/80 wt $\%$. W was lowest for film grafted by VAc and that grafted by EMA/VAc of $60 / 40 \mathrm{wt} \%$.

Acknowledgments. The authors thank Prof. Abo El-Khair B. M., Chemistry Department, University College for Women, Ain Shams University, and Prof. M. A. Ahmed, Faculty of Science, Cairo University, for valuable advices.

\section{REFERENCES}

1. N. G. McCrum, B. E. Read, and G. Williams, "Anelastic and Dielectric Effects in Polymeric Solids,” Wiley, London, 1967.

2. R. F. Boyer, J. Polym. Sci., C, 14, 3 (1966).

3. G. Williams, Advances in "Polymer Science, Vol. 33, SpringerVerlag Berlin Heidelberg, New York, 1979, pp 59-92.

4. P. Hedvig, "Dielectric Spectroscopy of Polymers," Adam Hilger Ltd., Bristol (Budapest, Hungary) 1977.

5. T. G. Parker, "Polymer Science," A. B. Jenkins, Ed., North-Holland, Publishing Company, Amsterdam, 1972, Chapter 19.

6. W. Reddish, Trans. Farady Soc., 46, 459 (1950).

7. K. Yamafuji, J. Phys. Soc. Jpn., 15, 2295 (1960).

8. Y. Ishida, Kolloid Z., 171, 71 (1960)

9. K. Yamafuji and Y. Ishida, Kolloid Z., 183, 15 (1962).

10. Y. Ishida, J. Polym. Sci., A-2, 7, 1835 (1969).

11. G. Williams and D. A. Edwards, Trans. Farady Soc., 62, 1329 (1966).

12. G. Williams, Trans. Farady Soc., 62, 2091 (1966).

13. H. Sasabe and S. Saito, J. Polym. Sci., A-2, 6, 1401 (1968).

14. H. Sasabe, "Reesarch of the Electrotechnichal Laboratories Tokyo, Japan," The Electro-Technical Laboratories, Tokyo, Publication 721 (1971).

15. Y. Ishida, M. Matsuo, and K. Yama Loji, Kolloid Z., 180, 108 (1962). 
16. K. Hikichi and J. Furichi, Rept. Progr. Polym. Phys. Jpn., 4, 69 (1961).

17. S. Saito, S. Hiroyuki, and N. Tatsuji, J. Polym. Sci., A-2, 6, 1297 (1968).

18. Y. Kihira and H. Yamamura, J. Polym. Sci., B, 24, 867 (1986).

19. A. K. Gupta and N. Chand, J. Polym. Sci., Polym. Phys. Ed., 18, 1125 (1980)

20. G. S. Rellick and J. Runt, J. Polym. Sci., B, 26, 1425 (1988).

21. S. Yano, J. Polym. Sci., A-2, 8, 1057 (1970).

22. H. Sasabe, S. Saito, M. Asahina, and H. Kakutani, J. Polym. Sci., $A-2,7,1405$ (1969).

23. P. A. M. Steeman and F. H. J. Maurer, Polymer, 33, 4236 (1992).

24. C. Mai and G. P. Johuri, J. Polym. Sci., B, 25, 1903 (1987).

25. S. H. Chung, K. Pathmanathan, and G. P. Johari, J. Polym. Sci., Polym. Phys. Ed., 24, 2655 (1986).
26. I. Maxwell and R. A. Pethrick, J. Appl. Polym. Sci., 28, 2363 (1983).

27. T. J. Lewis, "Spec. Periodical Reports, Dielectric and Molecular Process," Vol. 3, M. M. Davis, Ed., The Chemical Society, 1977, London.

28. D. J. Croften and R. A. Pethrick, Polymer, 22, 1048 (1981).

29. A. Mansingh, J. M. Reyers, and M. Sayer, J. Non-Crystalline Solids, 7, 12 (1972).

30. M. Sayer, A. Mansingh, J. M. Reyes, and G. Rosenblatt, J. Appl. Phys., 42, 2857 (1971).

31. B. K. Chaudhuri, K. Chaudhuri, and K. K. Som, J. Phys. Chem. Solids, 50, 1149 (1989). 